\title{
Clinical management of alveolar osteitis. A systematic review
}

\author{
Federico Garola ${ }^{1}$, Gerardo Gilligan ${ }^{2}$, René Panico ${ }^{3}$, Nicolás Leonardi ${ }^{4}$, Eduardo Piemonte ${ }^{5}$ \\ ${ }^{1}$ DDS, Oral Medicine Department, Facultad de Odontología, Universidad Nacional de Córdoba, Argentina \\ ${ }^{2}$ DDS, PhD, Assistant Professor, Oral Medicine Department, Facultad de Odontología, Universidad Nacional de Córdoba, Ar- \\ gentina \\ ${ }^{3}$ DDS, PhD, Head Professor, Oral Medicine Department, Facultad de Odontología, Universidad Nacional de Córdoba, Argentina \\ ${ }^{4}$ DDS, Oral Medicine Department, Escuela de Odontología, Facultad de Ciencias de la Salud, Universidad Católica de Córdoba, \\ Argentina \\ ${ }^{5}$ DDS, PhD, Associate Professor, Oral Medicine Department, Facultad de Odontología, Universidad Nacional de Córdoba, Ar- \\ gentina
}

Correspondence:

Haya de la Torre $S N$

PA: 5000. Ciudad Universitaria

Córdoba, Argentina

federico.garola@mi.unc.edu.ar

Garola F, Gilligan G, Panico R, Leonardi N, Piemonte E. Clinical management of alveolar osteitis. A systematic review. Med Oral Patol Oral Cir Bucal. 2021 Nov 1;26 (6):e691-702.

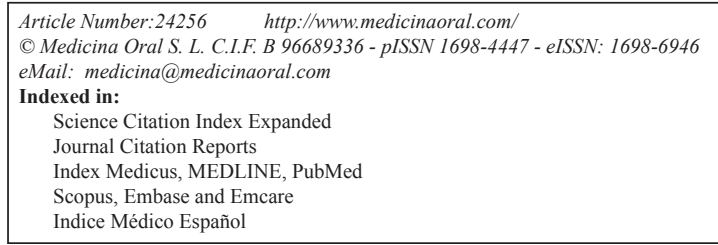

\begin{abstract}
Background: Alveolar Osteitis (AO) is one of the most common complications of tooth extraction. Several therapeutic interventions have been described for the treatment of $\mathrm{AO}$, however, there are no treatment standardized protocols. The aim of this study was to conduct a systematic review on the efficacy in pain control of the different treatments for AO. The feasibility of the application of these interventions is also discussed.

Material and Methods: A structured electronic and hand search strategy was applied to PubMed, Scopus, Cochrane Library, OpenGrey, and Google Scholar between January 2010 and July 2020 to identify studies according to PRISMA guidelines. The inclusion criteria were original English and Spanish clinical trials that analyzed paincontrol parameters according to visual analog scale (VAS, 0-10 scale), or pain relief patients' percentages. Those treatments that reach VAS $\leq 4$ on day 2 or before; or $\geq 85 \%$ of patients with absence of pain symptoms at day 7 or before were considered acceptable for their recommendation.

Results: The final review included 17 clinical trials. Among them, there were analyzed a total of 39 different AO treatments. 53,8\% of the treatments fulfill the proposed parameters for pain control.

Conclusions: Treatment alternatives are multiple, heterogeneous, and difficult to compare. The management of $\mathrm{AO}$ is summarized in basic (intra-alveolar irrigation) and specific procedures (Alveogyl ${ }^{\circledR}$, Neocones ${ }^{\circledR}$, SaliCept Patch ${ }^{\circledR}$, Low-Level Laser, Platelet-Rich Fibrin) that reach pain control success. They could be selected according to their availability and advantages or disadvantages.
\end{abstract}

Key words: Dry socket, alveolar osteitis, treatment, management, pain control, pain relief. 


\section{Introduction}

Alveolar osteitis (AO), localized osteitis, or usually so-called dry socket, is one of the most common complications of tooth extraction (1), with a frequency of 1 to $5 \%$ (2). In $30 \%$ of the cases, $\mathrm{AO}$ is frequently associated with extractions of mandibular third molars (3). AO is defined by the presence of postoperative pain in and around the post-extraction site, which increases in intensity between 1 and 3 days after extraction, accompanied by a partially or totally disintegrated blood clot within the alveolar socket, with or without evident halitosis (4). Regarding AO etiopathogenesis, it was described as partial or total fibrinolysis, possibly triggered by direct (physiological) or indirect (non-physiological) activating substances. After surgical trauma, alveolar bone cells release direct activators, while indirect activators are secreted by bacteria (5). Consequently, a necrotic socket in the absence of blood vessels and granulation tissue could alter alveolar healing.

Risk factors for $\mathrm{AO}$ are associated with difficult or traumatic extractions, female gender, tobacco use, oral contraceptive use, and pre-existing infection at the extraction site. The incidence of $\mathrm{AO}$ could be reduced by controlling these factors. Local application of chlorhexidine could be also useful (6).

Several therapeutic interventions have been described for the treatment of AO. In the 60s, the first reports of AO described the placement of zinc oxide (7). New therapeutic approaches were developed during the last decades. Among them, platelet-rich fibrin (PRF) is currently widely used in AO cases $(8,9)$. However, there are no treatment standardized protocols. The aim of this study was to perform a systematic review of the AO treatment considering pain control parameters and feasibility of application. The analysis of these data could be the first step to design a clinical guide for the management of $\mathrm{AO}$ by general dentists.

\section{Material and Methods}

A systematic review was performed in accordance with PRISMA declaration (10) (Preferred Reporting Items for Systematic Review and Meta-Analysis) to gather available and current evidence of $\mathrm{AO}$ treatment.

- Search strategy

Comprehensive electronic searches between January 2010 and July 2020 were performed. The searches were conducted in the following electronic databases: Pubmed-Medline, Scopus, and Cochrane Library. For searching the grey literature, OpenGrey and Google Scholar were also assessed. In addition, hand searches of relevant journals, such as those listed in other systematic reviews, were performed.

The search strategies for each database were as follow: In Cochrane Library, the search was performed using the following keywords: (dry socket treatment) or (dry socket management) or (alveolar osteitis treatment) or (alveolar osteitis management) or (treatment of dry socket) [all fields] [content type: trials]. In PubMed, the search was performed using the following keywords: (dry socket treatment) or (dry socket management) or (alveolar osteitis treatment) or (alveolar osteitis management) or (treatment of dry socket) and (trial) [title/abstract]. In Scopus, the search was performed using the following keywords: (dry socket treatment) or (dry socket management) or (alveolar osteitis treatment) or (alveolar osteitis management) or (treatment of dry socket) (limit-to (exactkeyword, "human")) or (limit-to (exactkeyword, "humans")) [all fields]. In Google Scholar, the search was performed using the following keywords: (management of dry socket) (without the words (preventive)) [in the title of the article]. In Open Grey, the search was performed using the following keywords: (dry socket treatment) OR (dry socket management) or (alveolar osteitis treatment) or (alveolar osteitis management) or (treatment of dry socket).

- Eligibility criteria

The present review focused on the following research question: What is the optimal clinical management for alveolar osteitis? PICOS (Population, Intervention, Comparison, Outcome, and Studies) schema for all the included studies to elaborate upon this research question were used to establish the eligibility criteria as follows:

Population: Adults patients with diagnosis of AO.

Intervention: Treatment or management of AO by intraalveolar clinical procedures.

Comparison: Other treatment or management of $\mathrm{AO}$ by intra-alveolar clinical procedures or absence of procedures.

Outcome: Pain level after treatment of AO by intra-alveolar clinical procedures.

Studies: Randomized and non-randomized clinical trials.

\section{- Inclusion criteria}

The search strategy was restricted to original English and Spanish languages. Inclusion criteria were clinical trials that analyzed pain-control parameters according to visual analog scale (VAS, 0-10 scale) or pain relief patients' percentages.

- Exclusion criteria

Preventive treatments, AO treated solely by antibiotics, analgesic-opiates or oral mouthwashes administration, deficient clinical data, lack of data regarding pain control, or records whose categorization was not adapted to the inclusion criteria were not included after full-text reading.

- Data extraction

Two independent researchers (F.G and G.G) conducted data extraction and validity assessment of the studies 
that met the inclusion criteria. Any discrepancy between the researchers was discussed with a third researcher (E.P) until consensus was reached. Relevant information for each study was entered into a predesigned data extraction form.

- Risk of bias in individual studies

The risk of bias of the included randomized controlled trials (RCTs) was assessed by the Cochrane Risk of Bias (RoB) tool (11). "High", "low," or "unclear" risk scores were based on the randomization method, allocation concealment, blinding of participants, personnel, and outcome assessors, completeness of outcome data, selective reporting, and other bias. Then, the overall risk of bias for each study was reported using the following criteria:

Low risk of bias: all domains are judged to be at low risk of bias.

Unclear risk of bias: one or more domains judged to be at unclear risk of bias.

High risk of bias: one or more domains judged to be at high risk of bias.

The risk of bias in non-randomized studies of interventions (ROBINS-I) (12) was used to assess the non-RCTs included. This tool evaluates the following domains: bias due to confounding, bias in selection of participants into the study, bias in classification of interventions, bias due to deviations from intended interventions, bias due to missing data, bias in measurement of outcomes, and bias in selection of the reported result. Finally, the overall risk of bias for each study was reported using the following criteria:

Low risk of bias: if the study was at low risk of bias for all domains.

Moderate risk of bias: if the study was at low or moderate risk of bias for all domains.

Serious risk of bias: if the study was at serious risk of bias in at least one domain, but not at critical risk of bias in any domain.

Critical risk of bias: if the study was at critical risk of bias in at least one domain.

No information on which to base a judgment about risk of bias: if there was a lack of information in one or more key domains of bias.

The reviewers compared evaluations, resolved disagreements by consensus, and reported their assessments using Review Manager software (RevMan 5.4, The Nordic Cochrane Centre, Copenhagen, Denmark) for RCTs. Robvis tool (visualization tool for risk of bias assessments in a systematic review) was used for presenting the non-RCTs data as appropriate.

- Type of treatments

Treatments were classified as invasive or non-invasive due to the different nature and heterogenicity of the included studies. Invasive treatments were considered when the treatment procedure included bone curettage or suture, while the non-invasive ones did not perform these measures, whether or not they needed local anesthesia. Subsequently, treatments were also categorized as high and low complexity. High complexity treatment was considered when specific equipment and training were required (such as laser or PRF).

- Summary measures

Those treatments which showed pain reduction according to VAS of at least 4 (on a scale of 0 to 10 , with 10 being the highest pain score) in the 48 hours after the first session, were considered recommended. Likewise, those treatments that reach the average percentage of patients with absence of pain symptoms during a week were also considered acceptable for this review.

A meta-analysis was not performed due to heterogeneity of the results and the lack of measures of dispersion.

\section{Results}

In the initial database search, 355 records were identified, out of which 114 were eliminated because were duplicates. After the first screening, 201 records were excluded because they had no direct relationship with the subject or they were not clinical trials. Thus, 40 records were eligible for full-text reading; of these, 1 was a retracted article, 1 was removed for double publication, 2 were not in English or Spanish language, 2 were non-indexed articles, 4 did not register pain control as a variable as well as 13 others studies had lack of data regarding pain control or records whose categorization was not adapted to the inclusion criteria. Finally, there were included 17 studies $(8,9,13-27)$. Fig. 1 shows the flowchart of the systematic review search process. Among them, there were analyzed 1138 patients with AO and 39 different treatment protocols.

Table 1 summarizes the included studies in this review. - Type of treatments

$56.4 \%$ of AO treatments were considered as non-invasive. Among them, 81,8\% were classified as low complexity ones, while $18,2 \%$ were classified as high complexity. The remaining $43.6 \%$ of $\mathrm{AO}$ treatments were considered as invasive. Among them, 64,7\% were classified as low complexity ones, while $35,3 \%$ were classified as high complexity.

- Risk of bias across studies

Among the 12 RCTs included studies (8,13,15-19,22-25, $27), 8$ studies $(15,17,18,22-25,27)$ were classified as unclear and 4 studies $(8,13,16,19)$ were classified as high risk. The most frequent domain causing downgrading was allocation concealment. The risk of bias summary for RCTs is shown in Fig. 2.

The 5 non-RCTs included studies $(9,14,20,21,26)$ were classified as serious risk. The domains that most frequently caused downgrading were bias due to confounding and bias in the measurement of outcomes. The risk of bias summary for non-RCTs is shown in Fig. 3. 


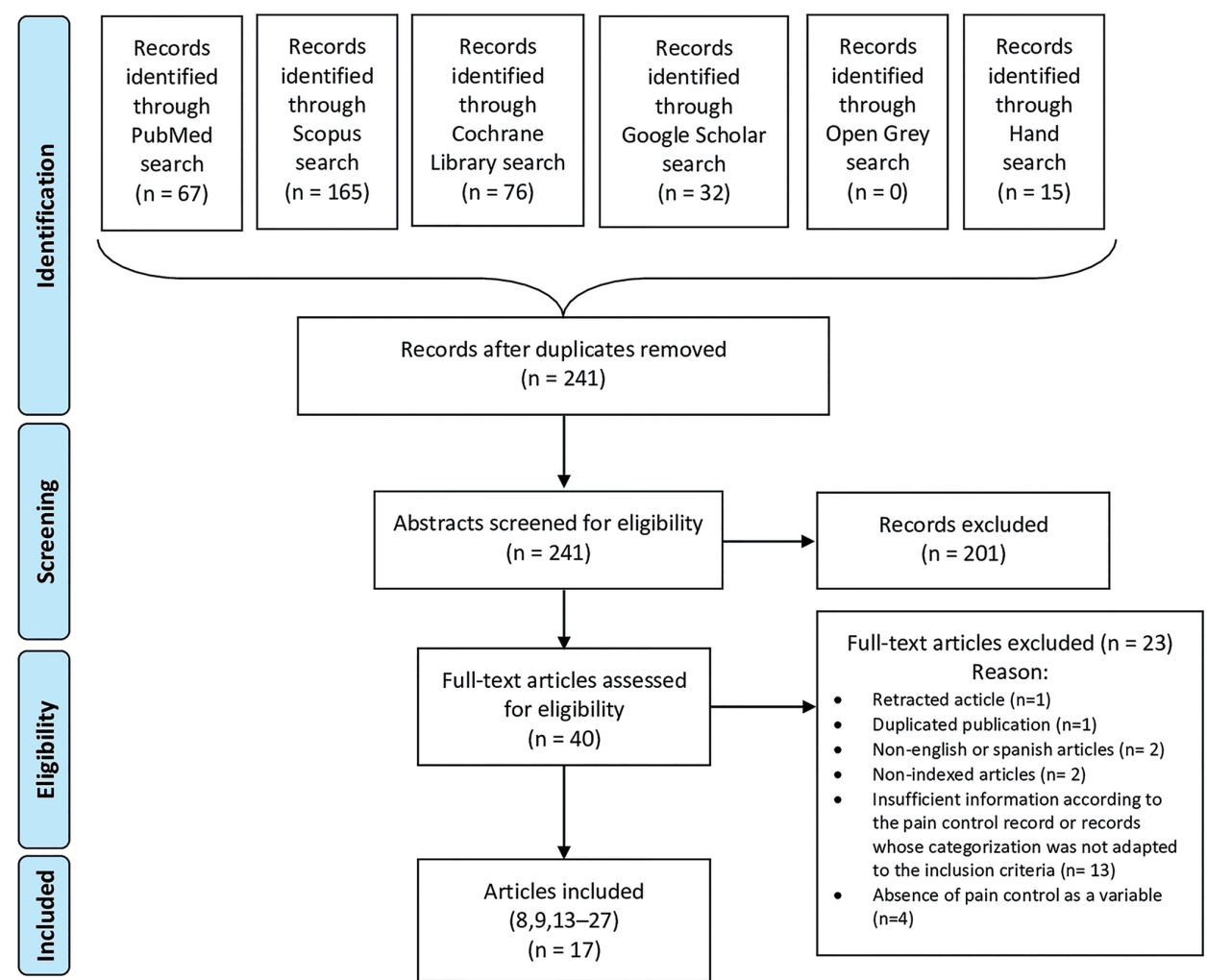

Fig. 1: PRISMA flowchart of systematic review search process.

Table 1: Summary of included randomized and non-randomized clinical trials.

\begin{tabular}{|c|c|c|c|c|}
\hline $\begin{array}{l}\text { Publica- } \\
\text { tion }\end{array}$ & $\begin{array}{l}\text { Study } \\
\text { type } \\
\text { (n) }\end{array}$ & Distribution & Treatment & Results \\
\hline $\begin{array}{l}\text { Kamal et } \\
\text { al. }(8) \\
2020\end{array}$ & $\begin{array}{l}\text { RCT } \\
(40)\end{array}$ & Not reported & $\begin{array}{l}\text { 1. Anesthesia, curettage, and sterile saline solution ir- } \\
\text { rigation } \\
\text { 2. Anesthesia, curettage, sterile saline solution irriga- } \\
\text { tion, PRF, and suture }\end{array}$ & $\begin{array}{l}\text { Immediate and delayed postop- } \\
\text { erative pain control was better in } \\
\text { patients who received PRF }\end{array}$ \\
\hline $\begin{array}{c}\text { Cebi (13) } \\
2020\end{array}$ & $\begin{array}{r}\mathrm{RCT} \\
(54)\end{array}$ & $\begin{array}{l}90,7 \% \text { mandibular } \\
\text { posterior region } \\
9,3 \% \text { maxillary poste- } \\
\quad \text { rior region }\end{array}$ & $\begin{array}{l}\text { 1. Curettage, sterile saline solution irrigation, Alveo- } \\
\text { gyl@ every } 2 \text { days during } 10 \text { days. Dexketoprofen } \\
\text { medication. Mouthwash with chlorhexidine } \\
\text { 2. Curettage, rifampicin irrigation, Alveogyl }{ }^{\circledR} \text { every } \\
2 \text { days during } 10 \text { days. Dexketoprofen medication. } \\
\text { Mouthwash with chlorhexidine } \\
\text { 3. Curettage, clindamycin irrigation, Alveogyl@ every } \\
2 \text { days during } 10 \text { days. Dexketoprofen medication. } \\
\text { Mouthwash with chlorhexidine }\end{array}$ & $\begin{array}{l}\text { Postoperative pain between } 4 \text { and } \\
5 \text { days was less in the group of } \\
\text { patients treated with clindamycin } \\
\text { irrigation }\end{array}$ \\
\hline $\begin{array}{l}\text { Suchánek } \\
\text { et al. }(14) \\
2019\end{array}$ & $\begin{array}{l}\text { Non- } \\
\text { RCT } \\
(50)\end{array}$ & $\begin{array}{l}82,8 \% \text { mandibular poste- } \\
\text { rior region }(62,1 \% \text { man- } \\
\text { dibular third molar) } \\
13,8 \% \text { maxillary poste- } \\
\text { rior region }\end{array}$ & $\begin{array}{l}\mathrm{H} 2 \mathrm{O} 2 \text { solution irrigation, a "pharmacological device" } \\
\text { composed of hyaluronic acid and octenidine dihydro- } \\
\text { chloride, replaced every day for } 7 \text { days }\end{array}$ & $\begin{array}{l}\text { Immediate postoperative pain } \\
\text { decreased, increased at } 18 \text { postop- } \\
\text { erative hours, and then decreased } \\
\text { again until treatment was com- } \\
\text { pleted }\end{array}$ \\
\hline $\begin{array}{l}\text { Yüce et al. } \\
(15) \\
2019\end{array}$ & $\begin{array}{r}\mathrm{RCT} \\
(40)\end{array}$ & $\begin{array}{l}100 \% \text { mandibular third } \\
\text { molar }\end{array}$ & $\begin{array}{l}\text { 1. Anesthesia, curettage, and sterile saline solution ir- } \\
\text { rigation every } 2 \text { days during } 7 \text { days } \\
\text { 2. Anesthesia, curettage, sterile saline solution irriga- } \\
\text { tion, PRF, and suture }\end{array}$ & $\begin{array}{c}\text { The immediate postoperative pain } \\
\text { was significantly less in the PRF } \\
\text { placement group }\end{array}$ \\
\hline $\begin{array}{l}\text { King et al. } \\
(16) \\
2018\end{array}$ & $\begin{array}{l}\text { RCT } \\
(38)\end{array}$ & $\begin{array}{c}93 \% \text { posterior region } \\
\text { (without maxillary or } \\
\text { mandibular specification) }\end{array}$ & $\begin{array}{l}\text { 1. Anesthesia, sterile saline solution irrigation and Al- } \\
\text { veogyl }{ }^{\circ} \\
\text { 2. Anesthesia, sterile saline solution irrigation, PRF, and } \\
\text { suture }\end{array}$ & $\begin{array}{l}\text { There were no significant differ- } \\
\text { ences in pain control }\end{array}$ \\
\hline
\end{tabular}


Table 1 cont.: Summary of included randomized and non-randomized clinical trials.

\begin{tabular}{|c|c|c|c|c|}
\hline $\begin{array}{l}\text { Supe et al. } \\
\quad(17) \\
2018\end{array}$ & $\begin{array}{r}\mathrm{RCT} \\
(50)\end{array}$ & $\begin{array}{l}78 \% \text { mandibular poste- } \\
\text { rior region }(33,3 \% \text { man- } \\
\text { dibular third molar) } \\
22 \% \text { maxillary posterior } \\
\text { region }\end{array}$ & $\begin{array}{l}\text { 1. Iodopovidone and sterile saline solution irrigation, } \\
\text { Alveogyl®. Diclofenac medication } \\
\text { 2. Iodopovidone, sterile saline solution irrigation, zinc } \\
\text { oxide eugenol paste. Diclofenac medication }\end{array}$ & $\begin{array}{l}\text { The postoperative pain was less in } \\
\text { the Alveogyl@ placement group, as } \\
\text { well as the complete remission of } \\
\text { the painful symptoms was faster }\end{array}$ \\
\hline $\begin{array}{l}\text { Chaurasia } \\
\text { et al. }(18) \\
2017\end{array}$ & $\begin{array}{l}\mathrm{RCT} \\
(88)\end{array}$ & $\begin{array}{l}76 \% \text { mandibular poste- } \\
\text { rior region }(69,3 \% \text { man- } \\
\text { dibular third molar) } \\
20,4 \% \text { maxillary poste- } \\
\text { rior region }\end{array}$ & $\begin{array}{l}\text { 1. Sterile saline solution irrigation, zinc oxide eugenol } \\
\text { paste mixed with a cotton pellet, replaced every day } \\
\text { until the pain subsided } \\
\text { 2. Sterile saline solution irrigation, Alveogyl@ replaced } \\
\text { every day until the pain subsided }\end{array}$ & $\begin{array}{l}\text { Postoperative pain was less from } \\
\text { the } 1 \text { st to the } 7 \text { th day in the zinc } \\
\text { oxide-eugenol placement group }\end{array}$ \\
\hline $\begin{array}{l}\text { Lone et al. } \\
\text { (19) } \\
2017\end{array}$ & $\begin{array}{l}\text { RCT } \\
(178)\end{array}$ & Not reported & $\begin{array}{l}\text { 1. Sterile saline solution irrigation, turmeric, and mus- } \\
\text { tard oil } \\
\text { 2. Sterile saline solution irrigation, zinc oxide eugenol } \\
\text { paste }\end{array}$ & $\begin{array}{c}\text { There was a significant pain reduc- } \\
\text { tion in the turmeric and mustard oil } \\
\text { placement group }\end{array}$ \\
\hline $\begin{array}{l}\text { Rastogi } \text { et } \\
\text { al. }(20) \\
2017\end{array}$ & $\begin{array}{l}\text { Non- } \\
\text { RCT } \\
(100)\end{array}$ & $\begin{array}{l}73,3 \% \text { mandibular poste- } \\
\text { rior región } \\
25,7 \% \text { maxillary poste- } \\
\text { rior region }\end{array}$ & $\begin{array}{l}\text { Anesthesia, sterile saline solution irrigation, PRF, and } \\
\text { suture }\end{array}$ & $\begin{array}{l}\text { The immediate postoperative pain } \\
\text { was high, it decreased from the } 3 \mathrm{rd} \\
\text { day to the } 7 \text { th day where it ended }\end{array}$ \\
\hline $\begin{array}{l}\text { Chakra- } \\
\text { varthi (9) } \\
2017\end{array}$ & $\begin{array}{l}\text { Non- } \\
\text { RCT } \\
(10)\end{array}$ & Not reported & $\begin{array}{l}\text { Anesthesia, sterile saline solution irrigation, PRF, and } \\
\text { suture. Aceclofenac medication }\end{array}$ & $\begin{array}{c}\text { Pain scores decreased to } 1 \text { on the } \\
\text { first day except for one patient, and } \\
\text { scores decreased to } 0 \text { in all patients } \\
\text { after } 48 \text { hours }\end{array}$ \\
\hline $\begin{array}{l}\text { Nikita } \text { et } \\
\text { al. }(21) \\
2016\end{array}$ & $\begin{array}{l}\text { Non- } \\
\text { RCT } \\
(47)\end{array}$ & $\begin{array}{l}83 \% \text { mandibular poste- } \\
\text { rior región ( } 29,7 \text { mandi- } \\
\text { bular third molar) } \\
17 \% \text { maxillary posterior } \\
\text { region }\end{array}$ & $\begin{array}{l}\text { Cleaning the affected socket with sterile cotton pellets, } \\
\text { sterile saline solution irrigation, and sterile cotton mixed } \\
\text { with pure nature honey }\end{array}$ & $\begin{array}{c}\text { Immediate postoperative pain was } \\
\text { high, decreasing from the second } \\
\text { day to a } 90 \% \text { reduction on the fifth } \\
\text { day }\end{array}$ \\
\hline $\begin{array}{l}\text { Dubovina } \\
\text { et al. }(22) \\
2016\end{array}$ & $\begin{array}{l}\mathrm{RCT} \\
(60)\end{array}$ & Not reported & $\begin{array}{l}\text { 1. Sterile saline solution irrigation and hyaluronic acid } \\
\text { 2. Sterile saline solution irrigation, hyaluronic acid, and } \\
\text { aminocaproic acid } \\
\text { 3. Sterile saline solution irrigation and Alveogyl® } \\
\text { 4. Anesthesia, curettage, and hyaluronic acid } \\
\text { 5. Anesthesia, curettage, hyaluronic acid, and aminoca- } \\
\text { proic acid } \\
\text { 6. Anesthesia, curettage, and Alveogyl@ }\end{array}$ & $\begin{array}{l}\text { The use of hyaluronic acid, with } \\
\text { aminocaproic acid associated with } \\
\text { a previous curettage, showed a } \\
\text { faster reduction of pain, as well as } \\
\text { edema, halitosis, and lymphade- } \\
\text { nopathy, in comparison with the } \\
\text { use of Alveogyl® }\end{array}$ \\
\hline $\begin{array}{l}\text { Rani et al. } \\
\quad(23) \\
2015\end{array}$ & $\begin{array}{l}\mathrm{RCT} \\
(60)\end{array}$ & Not reported & $\begin{array}{l}\text { 1. Anesthesia, sterile saline solution irrigation, and Al- } \\
\text { veogyl®. Paracetamol medication } \\
\text { 2. Anesthesia, sterile saline solution irrigation, and low- } \\
\text { level diode laser. Paracetamol medication } \\
\text { 3. Anesthesia, sterile saline solution irrigation, and low- } \\
\text { level Er; Cr: YSGG laser. Paracetamol medication }\end{array}$ & $\begin{array}{l}\text { Pain control was better in groups } \\
\text { with laser treatment. Diode laser } \\
\text { irradiation showed the best perfor- } \\
\text { mance }\end{array}$ \\
\hline $\begin{array}{l}\text { Esh- } \\
\text { ghpour et } \\
\text { al. }(24) \\
2015\end{array}$ & $\begin{array}{r}\mathrm{RCT} \\
(60)\end{array}$ & $\begin{array}{l}100 \% \text { mandibular third } \\
\text { molar }\end{array}$ & $\begin{array}{l}\text { 1. Anesthesia, sterile saline solution irrigation, and Al- } \\
\text { veogyl@ every } 2 \text { days during } 7 \text { days } \\
\text { 2. Low power red InGaAlP laser for } 3 \text { consecutive days } \\
\text { 3. Low power infrared GaAlAs laser for } 3 \text { consecutive } \\
\text { days }\end{array}$ & $\begin{array}{l}\text { The placement of Alveogyl® } \\
\text { proved to be more effective in early } \\
\text { pain reduction. Late pain control } \\
\text { (from the second day) was better in } \\
\text { patients who received low power } \\
\text { red laser therapy }\end{array}$ \\
\hline $\begin{array}{l}\text { Faizel } \text { et } \\
\text { al. }(25) \\
2014\end{array}$ & $\begin{array}{l}\text { RCT } \\
(105)\end{array}$ & $\begin{array}{l}63,2 \% \text { mandibular poste- } \\
\text { rior region }(6,9 \% \text { mandi- } \\
\text { bular third molar) } \\
36,8 \% \text { maxillary poste- } \\
\text { rior region }\end{array}$ & $\begin{array}{l}\text { 1. Sterile saline solution irrigation, Alveogyl® } \\
\text { 2. Sterile saline solution irrigation, gauze piece soaked } \\
\text { with zinc oxide eugenol paste } \\
\text { 3. Sterile saline solution irrigation, Neocones }{ }^{\circledR}\end{array}$ & $\begin{array}{l}\text { The use of Neocones }{ }^{\circledR} \text { was better } \\
\text { in reducing the painful symptoms } \\
\text { over time. The healing was faster } \\
\text { and, therefore, the postsurgical } \\
\text { consultations were less }\end{array}$ \\
\hline $\begin{array}{l}\text { Singh } \text { et } \\
\text { al. }(26) \\
2014\end{array}$ & $\begin{array}{l}\text { Non- } \\
\text { RCT } \\
(54)\end{array}$ & Not reported & Sterile gouge soaked with pure nature honey & $\begin{array}{c}\text { Inflammation, erythema, exudate } \\
\text { and pain were reduced. There were } \\
\text { no allergic reactions or adverse } \\
\text { effects }\end{array}$ \\
\hline $\begin{array}{l}\text { Kaya et al. } \\
(27) \\
\mathbf{2 0 1 1}\end{array}$ & $\begin{array}{l}\mathrm{RCT} \\
(104)\end{array}$ & $\begin{array}{l}100 \% \text { mandibular poste- } \\
\text { rior region }\end{array}$ & $\begin{array}{l}\text { 1. Anesthesia, curettage and sterile saline solution irri- } \\
\text { gation every day for } 3 \text { days } \\
\text { 2. Anesthesia, curettage, sterile saline solution irriga- } \\
\text { tion and Alveogyl® every day for } 3 \text { days } \\
\text { 3. Anesthesia, curettage, sterile saline solution irriga- } \\
\text { tion and SaliCept Patch } ® \text { every day for } 3 \text { days } \\
\text { 4. Anesthesia, curettage,sterile saline solution irrigation } \\
\text { and low level GaAlAs laser every day for } 3 \text { days }\end{array}$ & $\begin{array}{l}\text { The treatment with low-level laser } \\
\text { therapy obtained the best perfor- } \\
\text { mance regarding pain control, } \\
\text { erythema, inflammation, halitosis } \\
\text { and exposed bone }\end{array}$ \\
\hline
\end{tabular}

n: alveolar osteitis treated patients, who completed the study; RCT: randomized controlled trial; PRF: platelet rich fibrin 


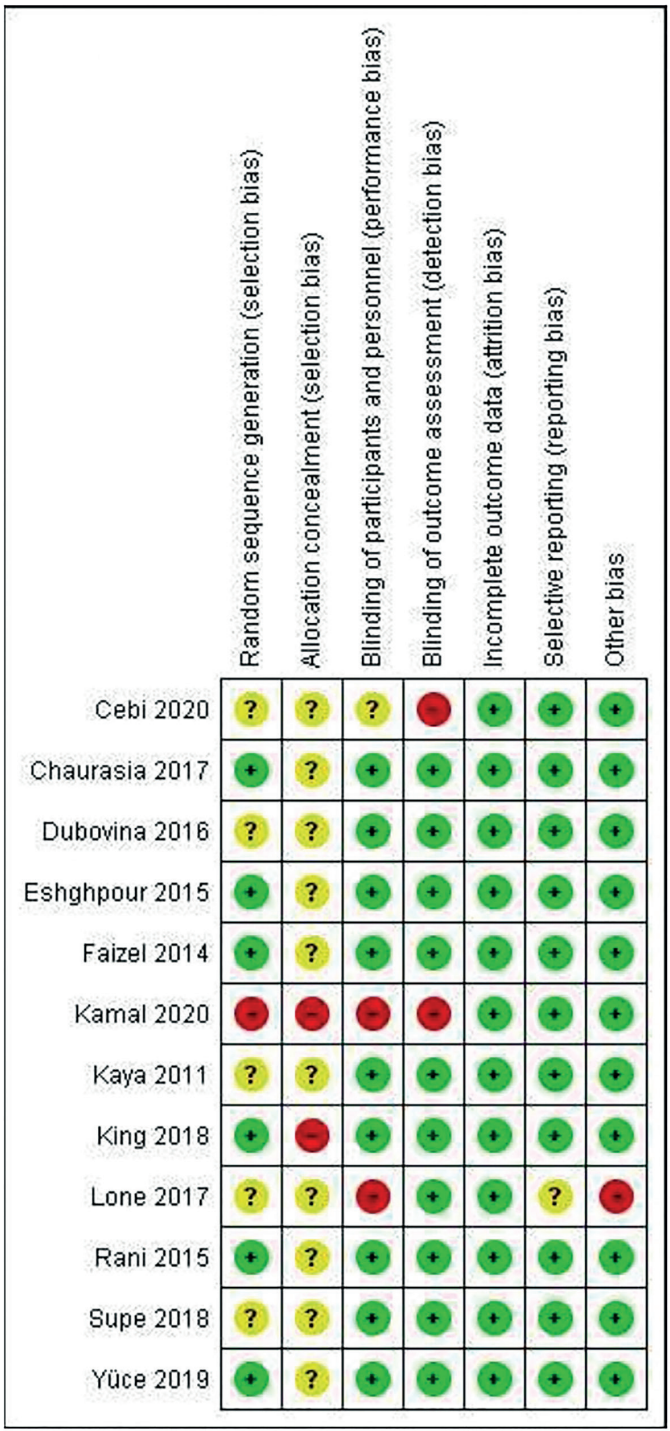

Fig. 2: Risk of bias summary for randomized controlled trials, assessed using the Cochrane Risk of Bias (RoB) tool: review authors' judgments about each risk of bias item for each included study $(+=$ low; $-=$ high;? = unclear $)$.

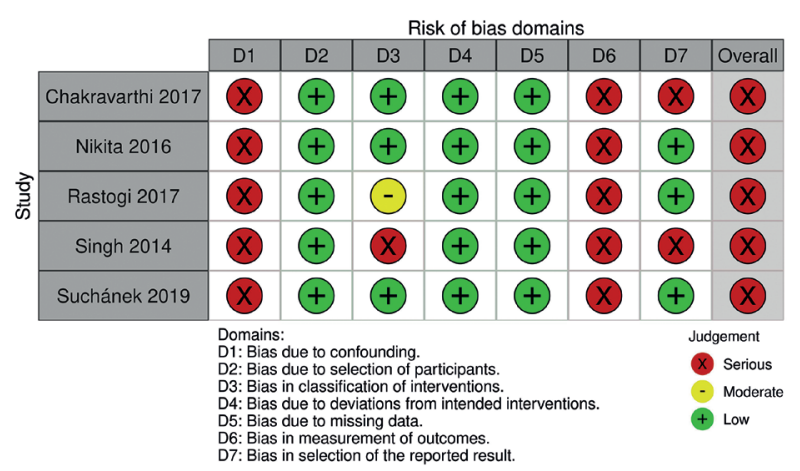

Fig. 3: Risk of bias summary for non-randomized controlled trials, assessed using The Risk Of Bias In Non-randomized Studies of Interventions ROBINS-I tool.
- Pain control

Table 2 shows pain control registered using VAS and pain relief patient's percentage registered in a 7-8-day period from the first consultation (day 0 ). The average of the percentages of patients without pain at one week of each treatment was $85 \%$. This value was considered as a cut-off point of recommendation. $53,8 \%$ of the treatments fulfill the proposed parameters for pain control.

- First visit clinical procedures and total sessions required

Table 3 shows the characteristics of alveolar osteitis treatments, comparing the number of clinical procedures performed at the first visit, the number of total sessions required during a week (suture removal was considered as a session, check-up visits without clinical interventions were not considered as a session), and administrated medication.

$58.9 \%$ of the treatments required at least three clinical procedures in the first visit and $69,2 \%$ of the treatments required at least three total treatment sessions.

- Pain control and total number of sessions

Comparing tables that evaluate pain control with the treatments that require fewer total sessions, the results (best performance treatments) are described below:

1) Low complexity non-invasive treatments, with fewer sessions required, that fulfill the proposed parameters for pain reduction:

Sterile saline solution irrigation and placement of Alveogyl ${ }^{\circledR}$. Three treatment sessions required (25).

Sterile saline solution irrigation and placement of Neocones ${ }^{\circledR}$. Two treatment sessions required (25). This is the treatment that achieved the best pain control with fewer sessions required.

2) High complexity non-invasive treatments, with fewer sessions required, that fulfill the proposed parameters for pain reduction:

Anesthesia, sterile saline solution irrigation, and lowlevel diode laser irradiation. Anti-inflammatory analgesic medication with paracetamol. One treatment session required (23).

3) Low complexity invasive treatments, with fewer sessions required, that fulfill the proposed parameters for pain reduction:

Anesthesia, curettage, and sterile saline solution irrigation. Placement of SaliCept Patch ${ }^{\circledR}$. Three treatment sessions required (27).

4) High complexity invasive treatments, with fewer sessions required, that fulfill the proposed parameters for pain reduction:

Anesthesia, curettage, and irrigation with sterile saline solution. Placement of PRF and suture. Two treatment sessions required (8).

Anesthesia, irrigation with sterile saline solution, placement of PRF, and suture. Two treatment sessions required (20). 
Table 2: Pain control of the treatments according to Visual Analog Scale and pain relief patients' percentages.

\begin{tabular}{|c|c|c|c|c|c|c|c|c|c|c|}
\hline \multirow{2}{*}{ Publication } & \multirow{2}{*}{ Treatment group } & \multicolumn{9}{|c|}{ Day } \\
\hline & & 0 & 1 & 2 & 3 & 4 & 5 & 6 & 7 & 8 \\
\hline \multicolumn{11}{|c|}{ Pain according to Visual Analog Scale } \\
\hline \multirow{2}{*}{$\begin{array}{l}\text { Kamal et al. } \\
\text { (8) } 2020\end{array}$} & Anesthesia, curettage, and sterile saline solution & 8,6 & - & - & - & 5,1 & - & - & 3,0 & - \\
\hline & Anesthesia, curettage, sterile saline solution, PRF, suture & 8 & - & - & - & 0,3 & - & - & 0 & - \\
\hline \multirow{3}{*}{$\begin{array}{c}\text { Cebi (13) } \\
\mathbf{2 0 2 0}\end{array}$} & Curettage, sterile saline solution, Alveogyl@ & $\begin{array}{c}7,4 \\
(1,3) \\
\end{array}$ & $\begin{array}{c}6,5 \\
(0,7)\end{array}$ & \begin{tabular}{|c|}
5,9 \\
$(0,9)$ \\
\end{tabular} & $\begin{array}{c}5,5 \\
(0,8) \\
\end{array}$ & - & \begin{tabular}{|c|}
4,4 \\
$(1,6)$
\end{tabular} & - & $\begin{array}{c}1,7 \\
(0,6)\end{array}$ & - \\
\hline & Curettage, rifampicin, Alveogyl® & $\begin{array}{c}7,4 \\
(1,0) \\
\end{array}$ & $\begin{array}{c}5,4 \\
(0,6) \\
\end{array}$ & $\begin{array}{c}5,0 \\
(0,6)\end{array}$ & $\begin{array}{c}4,5 \\
(0,8) \\
\end{array}$ & - & $\begin{array}{c}3,9 \\
(1,2) \\
\end{array}$ & - & $\begin{array}{c}1,5 \\
(1,0)\end{array}$ & - \\
\hline & Curettage, clindamycin, Alveogyl® & $\begin{array}{c}7,4 \\
(1,1) \\
\end{array}$ & $\begin{array}{c}4,8 \\
(1,1)\end{array}$ & $\begin{array}{c}4,5 \\
(1,0) \\
\end{array}$ & $\begin{array}{c}4,0 \\
(1,0)\end{array}$ & - & $\begin{array}{c}3,5 \\
(1,0)\end{array}$ & - & $\begin{array}{c}1,2 \\
(0,4)\end{array}$ & - \\
\hline $\begin{array}{l}\text { Suchánek et } \\
\text { al. (14) } 2019\end{array}$ & $\mathrm{H} 2 \mathrm{O} 2$ solution, pharmacological device & $\begin{array}{c}6,9 \\
(2,0)\end{array}$ & $\begin{array}{c}5 \\
(2,5)\end{array}$ & $\begin{array}{c}\mathbf{3 , 5} \\
(2,5) \\
\end{array}$ & $\begin{array}{c}2 \\
(2,0)\end{array}$ & $\begin{array}{c}1 \\
(1,6)\end{array}$ & $\begin{array}{c}0,6 \\
(1,3) \\
\end{array}$ & \begin{tabular}{|c|}
0,2 \\
$(0,9)$ \\
\end{tabular} & - & - \\
\hline \multirow{2}{*}{$\begin{array}{l}\text { Yüce et al. } \\
\text { (15) } 2019\end{array}$} & Anesthesia, curettage, and sterile saline solution & $\begin{array}{c}6,8 \\
(0,8) \\
\end{array}$ & $\begin{array}{c}7,2 \\
(1,0) \\
\end{array}$ & - & $\begin{array}{c}7,0 \\
(1,2) \\
\end{array}$ & - & \begin{tabular}{|c|}
5,9 \\
$(0,8)$ \\
\end{tabular} & - & \begin{tabular}{|c|}
4,0 \\
$(0,7)$ \\
\end{tabular} & - \\
\hline & Anesthesia, curettage, sterile saline solution, PRF, and suture & $\begin{array}{c}7,1 \\
(1,0) \\
\end{array}$ & $\begin{array}{c}5,2 \\
(1,0)\end{array}$ & - & $\begin{array}{c}2,2 \\
(0,6) \\
\end{array}$ & - & $\begin{array}{c}0,8 \\
(0,6)\end{array}$ & - & $\begin{array}{c}0,4 \\
(0,5) \\
\end{array}$ & - \\
\hline \multirow{2}{*}{$\begin{array}{l}\text { King et al. } \\
\text { (16) } 2018\end{array}$} & Anesthesia, sterile saline solution and Alveogyl® & $\begin{array}{c}6,6 \\
(2,3) \\
\end{array}$ & - & - & \begin{tabular}{|c|}
4,3 \\
$(2,9)$ \\
\end{tabular} & - & - & - & $\begin{array}{c}2,4 \\
(2,6) \\
\end{array}$ & - \\
\hline & Anesthesia, sterile saline solution, PRF, and suture & $\begin{array}{c}6,4 \\
(1,8) \\
\end{array}$ & - & - & \begin{tabular}{|c|}
4,0 \\
$(2,7)$ \\
\end{tabular} & - & - & - & $\begin{array}{c}2,0 \\
(2,0) \\
\end{array}$ & - \\
\hline \multirow{2}{*}{$\begin{array}{l}\text { Supe et al. } \\
\text { (17) } 2018\end{array}$} & Iodopovidone and sterile saline solution, Alveogyl® & $\begin{array}{c}8,4 \\
(1,0) \\
\end{array}$ & - & - & $\begin{array}{c}3,9 \\
(1,5)\end{array}$ & - & $\begin{array}{c}1,6 \\
(1,5)\end{array}$ & - & $\begin{array}{c}0,4 \\
(0,9) \\
\end{array}$ & - \\
\hline & Iodopovidone, sterile saline solution, Zinc oxide eugenol paste & $\begin{array}{c}8,9 \\
(0,7) \\
\end{array}$ & - & - & $\begin{array}{c}5,8 \\
(1,8) \\
\end{array}$ & - & $\begin{array}{c}3,6 \\
(1,8) \\
\end{array}$ & - & $\begin{array}{c}2,5 \\
(1,7)\end{array}$ & - \\
\hline \multirow{2}{*}{$\begin{array}{l}\text { Chaurasia et } \\
\text { al. (18) } 2017\end{array}$} & $\begin{array}{l}\text { Sterile saline solution, Zinc oxide eugenol paste mixed with a } \\
\text { cotton pellet }\end{array}$ & $\begin{array}{c}7,5 \\
(1,0) \\
\end{array}$ & - & \begin{tabular}{|c|}
$\mathbf{2 , 8}$ \\
$(1,1)$ \\
\end{tabular} & - & - & \begin{tabular}{|c|}
1,4 \\
$(0,9)$ \\
\end{tabular} & - & $\begin{array}{c}0,5 \\
(0,6) \\
\end{array}$ & - \\
\hline & Sterile saline solution, Alveogyl® & $\begin{array}{c}7,6 \\
(1,2)\end{array}$ & - & $\begin{array}{c}3,4 \\
(0,9) \\
\end{array}$ & - & - & \begin{tabular}{c|c|}
2,2 \\
$(0,7)$
\end{tabular} & - & $\begin{array}{l}1,1 \\
(0,5) \\
\end{array}$ & - \\
\hline $\begin{array}{l}\text { Rastogi et al. } \\
(20) 2017\end{array}$ & Anesthesia, sterile saline solution, PRF, and suture & $\begin{array}{c}8,5 \\
(0,5) \\
\end{array}$ & $\begin{array}{c}4,5 \\
(0,6) \\
\end{array}$ & - & \begin{tabular}{|c|}
2,3 \\
$(0,4)$ \\
\end{tabular} & - & - & - & $\begin{array}{c}0 \\
(0,0) \\
\end{array}$ & - \\
\hline $\begin{array}{l}\text { Chakravarthi } \\
\text { (9) } 2017\end{array}$ & Anesthesia, sterile saline solution, PRF and suture & 6,8 & 1,1 & $\mathbf{0 , 1}$ & 0 & - & - & - & - & - \\
\hline $\begin{array}{l}\text { Nikita et al. } \\
\text { (21) } 2016\end{array}$ & $\begin{array}{l}\text { Cleaning the socket with sterile cotton pellets, sterile saline } \\
\text { solution, and cotton mixed with pure nature honey }\end{array}$ & $\begin{array}{c}7,3 \\
(1,1)\end{array}$ & $\begin{array}{c}4,7 \\
(1,1) \\
\end{array}$ & $\begin{array}{c}\mathbf{2 , 2} \\
(0,8) \\
\end{array}$ & - & \begin{tabular}{|c|}
0,7 \\
$(0,5)$ \\
\end{tabular} & - & - & - & - \\
\hline \multirow{6}{*}{$\begin{array}{l}\text { Dubovina et } \\
\text { al. }(22) 2016\end{array}$} & Sterile saline solution and hyaluronic acid & $\begin{array}{c}7,3 \\
(2,0) \\
\end{array}$ & - & $\begin{array}{c}5,1 \\
(2,5) \\
\end{array}$ & - & \begin{tabular}{|c|}
2,4 \\
$(2,1)$ \\
\end{tabular} & - & $\begin{array}{c}0,7 \\
(1,1) \\
\end{array}$ & - & - \\
\hline & Sterile saline solution, hyaluronic aminocaproic acid & $\begin{array}{c}7,9 \\
(1,6) \\
\end{array}$ & - & $\begin{array}{c}5,1 \\
(2,5) \\
\end{array}$ & - & \begin{tabular}{|c|}
2,4 \\
$(2,1)$ \\
\end{tabular} & - & $\begin{array}{c}0,7 \\
(1,1) \\
\end{array}$ & - & - \\
\hline & Sterile saline solution and Alveogyl® & $\begin{array}{c}7,4 \\
(1,4) \\
\end{array}$ & - & $\begin{array}{c}7,2 \\
(1,3) \\
\end{array}$ & - & $\begin{array}{c}5,1 \\
(1,9) \\
\end{array}$ & - & $\begin{array}{c}2,9 \\
(1,9) \\
\end{array}$ & - & - \\
\hline & Anesthesia, curettage, and hyaluronic acid & $\begin{array}{c}7,7 \\
(1,4) \\
\end{array}$ & - & \begin{tabular}{|c|}
$\mathbf{3 , 8}$ \\
$(2,7)$ \\
\end{tabular} & - & $\begin{array}{c}1,6 \\
(1,5) \\
\end{array}$ & - & $\begin{array}{c}0,3 \\
(0,6) \\
\end{array}$ & - & - \\
\hline & Anesthesia, curettage, hyaluronic aminocaproic acid & $\begin{array}{c}7,9 \\
(1,5) \\
\end{array}$ & - & $\begin{array}{c}\mathbf{3 , 5} \\
(2,3) \\
\end{array}$ & - & \begin{tabular}{c|}
1,8 \\
$(1,8)$ \\
\end{tabular} & - & $\begin{array}{c}0,6 \\
(1,1) \\
\end{array}$ & - & - \\
\hline & Anesthesia, curettage, and Alveogyl® & $\begin{array}{c}7,4 \\
(1,6) \\
\end{array}$ & - & $\begin{array}{c}6,1 \\
(2,6) \\
\end{array}$ & - & \begin{tabular}{|c|}
4,3 \\
$(2,5)$ \\
\end{tabular} & - & $\begin{array}{c}2,1 \\
(1,9) \\
\end{array}$ & - & - \\
\hline \multirow{3}{*}{$\underset{\text { (23) } 2015}{\text { Rani et al. }}$} & Anesthesia, sterile saline solution and Alveogyl® & $\begin{array}{c}7,3 \\
(1,2) \\
\end{array}$ & $\begin{array}{c}5,5 \\
(1,5) \\
\end{array}$ & \begin{tabular}{|c|}
4,6 \\
$(1,6)$ \\
\end{tabular} & $\begin{array}{c}3,3 \\
(1,6) \\
\end{array}$ & \begin{tabular}{|c|}
2 \\
$(1,7)$ \\
\end{tabular} & \begin{tabular}{|c|}
1,5 \\
$(1,8)$ \\
\end{tabular} & \begin{tabular}{|c|}
1,9 \\
$(2,1)$ \\
\end{tabular} & - & - \\
\hline & Anesthesia, sterile saline solution and low-level diode laser & $\begin{array}{c}7 \\
(1,0) \\
\end{array}$ & $\begin{array}{c}5,2 \\
(1,7) \\
\end{array}$ & $\begin{array}{c}3,2 \\
(1,8) \\
\end{array}$ & $\begin{array}{c}1,5 \\
(1,6) \\
\end{array}$ & \begin{tabular}{|c|}
0,6 \\
$(0,8)$ \\
\end{tabular} & \begin{tabular}{|c|}
0,3 \\
$(0,6)$ \\
\end{tabular} & \begin{tabular}{|c|}
0,5 \\
$(0,5)$ \\
\end{tabular} & - & - \\
\hline & $\begin{array}{l}\text { Anesthesia, sterile saline solution and low-level Er; Cr:YSGG } \\
\text { laser }\end{array}$ & $\begin{array}{c}7 \\
(1,3) \\
\end{array}$ & \begin{tabular}{|c|}
4,7 \\
$(2,0)$ \\
\end{tabular} & $\begin{array}{c}3,7 \\
(1,8) \\
\end{array}$ & $\begin{array}{c}2,6 \\
(1,8) \\
\end{array}$ & \begin{tabular}{c|c|}
1,5 \\
$(1,4)$ \\
\end{tabular} & \begin{tabular}{|c|c|}
0,6 \\
$(1,1)$ \\
\end{tabular} & $\begin{array}{c}0,9 \\
(1,4) \\
\end{array}$ & - & - \\
\hline \multirow{3}{*}{$\begin{array}{l}\text { Eshghpour et } \\
\text { al. }(24) 2015\end{array}$} & Anesthesia, sterile saline solution and Alveogyl ${ }^{\circledR}$ & 7,8 & 2,1 & 3 & 0,4 & - & - & - & - & - \\
\hline & Low power red InGaAlP laser & 8,2 & 4,3 & 1,9 & 0,1 & - & - & - & - & - \\
\hline & Low power infrared GaAlAs laser & 8 & 5,2 & 4 & 1,1 & - & - & - & - & - \\
\hline \multicolumn{11}{|c|}{ Pain relief patient's percentage } \\
\hline & & & & $\%$ of pa & atients & withot & it pain & in Day & & \\
\hline \multirow{2}{*}{$\begin{array}{l}\text { Kamal et al. } \\
\text { (8) } 2020\end{array}$} & Anesthesia, curettage, and sterile saline solution & - & - & - & - & 0 & - & - & 6,6 & - \\
\hline & Anesthesia, curettage, sterile saline solution, PRF, and suture & - & - & - & - & 70 & - & - & 100 & - \\
\hline $\begin{array}{l}\text { Suchánek et } \\
\text { al. (14) } 2019\end{array}$ & $\mathrm{H} 2 \mathrm{O} 2$ solution, pharmacological device & - & - & - & 19 & 62 & - & - & 96 & - \\
\hline \multirow{2}{*}{$\begin{array}{l}\text { Lone et al. } \\
\text { (19) } 2017\end{array}$} & Sterile saline solution, turmeric mustard oil & - & - & 61 & 88 & 100 & - & - & - & - \\
\hline & Sterile saline solution, zinc oxide eugenol paste & - & - & 0 & 0 & 45 & 87 & - & 100 & - \\
\hline $\begin{array}{l}\text { Rastogi et al. } \\
\text { (20) } 2017\end{array}$ & Anesthesia, sterile saline solution, PRF, and suture & - & - & - & - & - & - & - & 100 & - \\
\hline
\end{tabular}


Table 2 cont.: Pain control of the treatments according to Visual Analog Scale and pain relief patients' percentages.

\begin{tabular}{|c|c|c|c|c|c|c|c|c|c|c|}
\hline $\begin{array}{l}\text { Chakravarthi } \\
\text { (9) } 2017\end{array}$ & Anesthesia, sterile saline solution, PRF, and suture & - & - & 90 & 100 & - & - & - & - & - \\
\hline $\begin{array}{l}\text { Nikita et al. } \\
\text { (21) } 2016\end{array}$ & $\begin{array}{l}\text { Cleaning the socket with sterile cotton pellets, sterile saline } \\
\text { solution, and sterile cotton mixed with pure nature honey }\end{array}$ & - & - & 35 & 70 & - & 90 & - & - & - \\
\hline \multirow{3}{*}{$\begin{array}{l}\text { Faizel et al. } \\
\text { (25) } 2014\end{array}$} & Sterile saline solution, Alveogyl® & - & - & - & - & - & - & $\begin{array}{c}\mathbf{1 0 0} \\
(0,4)\end{array}$ & - & - \\
\hline & $\begin{array}{l}\text { Sterile saline solution, gauze piece soaked with zinc oxide } \\
\text { eugenol paste }\end{array}$ & - & - & - & - & - & - & - & - & $\begin{array}{r}100 \\
(0,4) \\
\end{array}$ \\
\hline & Sterile saline solution, Neocones® & - & - & - & - & $\begin{array}{l}100 \\
(0,6)\end{array}$ & - & - & - & - \\
\hline $\begin{array}{l}\text { Singh } \text { et al. } \\
\text { (26) } 2014\end{array}$ & Sterile gouge soaked with pure nature honey & - & - & - & - & - & 55 & 85 & - & 100 \\
\hline \multirow{4}{*}{$\begin{array}{l}\text { Kaya et al. } \\
(27) 2011\end{array}$} & Anesthesia, curettage and sterile saline solution & - & - & - & 0 & - & - & - & 11 & - \\
\hline & Anesthesia, curettage, sterile saline solution and Alveogyl® & - & - & - & 20 & - & - & - & 76 & - \\
\hline & $\begin{array}{l}\text { Anesthesia, curettage, sterile saline solution and SaliCept } \\
\text { Patch® }\end{array}$ & - & - & - & 4 & - & - & - & 88 & - \\
\hline & $\begin{array}{l}\text { Anesthesia, curettage, sterile saline solution and low level } \\
\text { GaAlAs laser }\end{array}$ & - & - & - & 70 & - & - & - & 96 & - \\
\hline
\end{tabular}

In Pain according to Visual Analog Scale, bold indicates the fulfillment of pain reduction proposed parameters: VAS $\leq 4$ on day 2 or before.

Values are expressed as mean. SD-standard deviation is reported in parentheses.

In Pain relief patient's percentage, bold indicates the fulfillment of pain reduction proposed parameters: $\geq 85 \%$ of patients without pain at day 7 or before.

PRF: platelet rich fibrin.

Table 3: Characteristics of alveolar osteitis treatments and fulfillment of pain reduction proposed parameters.

\begin{tabular}{|c|c|c|c|c|c|c|c|}
\hline Publication & Treatment group & $\begin{array}{l}\text { Type of } \\
\text { treatment }\end{array}$ & $\begin{array}{c}\text { Number of } \\
\text { procedures } \\
\text { in the first } \\
\text { visit }\end{array}$ & $\begin{array}{l}\text { Sessions/ } \\
\quad \text { week }\end{array}$ & $\begin{array}{l}\text { Anti-inflam- } \\
\text { matory analgesic } \\
\text { medication }\end{array}$ & $\begin{array}{l}\text { Antibiotic } \\
\text { medication }\end{array}$ & $\begin{array}{l}\text { Fulfillment } \\
\text { of proposed } \\
\text { parameters }\end{array}$ \\
\hline \multirow{2}{*}{$\begin{array}{c}\text { Kamal et } \\
\text { al. }(8) \\
2020\end{array}$} & $\begin{array}{l}\text { Anesthesia, curettage, and sterile } \\
\text { saline solution irrigation }\end{array}$ & LC-I & 3 & 2 & No & No & No \\
\hline & $\begin{array}{l}\text { Anesthesia, curettage, sterile } \\
\text { saline solution irrigation. PRF } \\
\text { and suture }\end{array}$ & HC-I & 5 & 2 & No & No & Yes \\
\hline \multirow{3}{*}{$\begin{array}{l}\text { Cebi (13) } \\
\mathbf{2 0 2 0}\end{array}$} & $\begin{array}{l}\text { Curettage, sterile saline solution } \\
\text { irrigation and Alveogyl® }\end{array}$ & LC-I & 3 & 5 & $\begin{array}{l}\text { Yes Dexketo- } \\
\text { profen }\end{array}$ & No & No \\
\hline & $\begin{array}{l}\text { Curettage, rifampicin irrigation } \\
\text { and Alveogyl }{ }^{\circledR}\end{array}$ & LC-I & 3 & 5 & $\begin{array}{c}\text { Yes } \\
\text { Dexketoprofen }\end{array}$ & No & No \\
\hline & $\begin{array}{l}\text { Curettage, clindamycin irriga- } \\
\text { tion, and Alveogyl@ }\end{array}$ & LC-I & 3 & 5 & $\begin{array}{c}\text { Yes } \\
\text { Dexketoprofen }\end{array}$ & No & No \\
\hline $\begin{array}{l}\text { Suchánek } \\
\text { et al. }(14) \\
2019\end{array}$ & $\begin{array}{l}\mathrm{H} 2 \mathrm{O} 2 \text { solution irrigation and } \\
\text { pharmacological device }\end{array}$ & LC-NI & 2 & 7 & No & No & Yes \\
\hline \multirow{2}{*}{$\begin{array}{c}\text { Yüce et al. } \\
\text { (15) } \\
2019\end{array}$} & $\begin{array}{l}\text { Anesthesia, curettage, and sterile } \\
\text { saline solution irrigation }\end{array}$ & LC-I & 3 & 4 & No & No & No \\
\hline & $\begin{array}{l}\text { Anesthesia, curettage, sterile } \\
\text { saline solution irrigation, PRF, } \\
\text { and suture }\end{array}$ & HC-I & 5 & 2 & No & No & No \\
\hline \multirow{2}{*}{$\begin{array}{c}\text { King et al. } \\
\text { (16) } \\
\mathbf{2 0 1 8}\end{array}$} & $\begin{array}{l}\text { Anesthesia, sterile saline solution } \\
\text { irrigation and Alveogyl® }\end{array}$ & LC-NI & 3 & 1 & No & No & No \\
\hline & $\begin{array}{l}\text { Anesthesia, sterile saline solution } \\
\text { irrigation, PRF, and suture }\end{array}$ & HC-I & 4 & 2 & No & No & No \\
\hline \multirow{2}{*}{$\begin{array}{c}\text { Supe et al. } \\
\text { (17) } \\
2018\end{array}$} & $\begin{array}{l}\begin{array}{l}\text { Iodopovidone and sterile saline } \\
\text { solution irrigation and Alveogyl® }\end{array} \\
\end{array}$ & LC-NI & 2 & 2 & $\begin{array}{c}\text { Yes } \\
\text { Diclofenac } \\
\end{array}$ & No & No \\
\hline & $\begin{array}{l}\text { Iodopovidone and sterile saline } \\
\text { solution irrigation. Zinc oxide } \\
\text { eugenol paste }\end{array}$ & LC-NI & 2 & $3-4$ & $\begin{array}{c}\text { Yes } \\
\text { Diclofenac }\end{array}$ & No & No \\
\hline \multirow{2}{*}{$\begin{array}{l}\text { Chaurasia } \\
\text { et al. }(18) \\
2017\end{array}$} & $\begin{array}{l}\text { Sterile saline solution irrigation. } \\
\text { Zinc oxide eugenol paste mixed } \\
\text { with a cotton pellet }\end{array}$ & LC-NI & 2 & 7 & No & No & Yes \\
\hline & $\begin{array}{l}\text { Sterile saline solution irrigation } \\
\text { and Alveogyl }{ }^{\circledR}\end{array}$ & LC-NI & 2 & 7 & No & No & Yes \\
\hline
\end{tabular}


Table 3 cont.: Characteristics of alveolar osteitis treatments and fulfillment of pain reduction proposed parameters.

\begin{tabular}{|c|c|c|c|c|c|c|c|}
\hline \multirow{2}{*}{$\begin{array}{l}\text { Lone et al. } \\
\quad(19) \\
2017\end{array}$} & $\begin{array}{l}\text { Sterile saline solution irrigation. } \\
\text { Turmeric and mustard oil }\end{array}$ & LC-NI & 2 & 4 & No & No & Yes \\
\hline & $\begin{array}{l}\text { Sterile saline solution irrigation } \\
\text { and zinc oxide eugenol paste }\end{array}$ & LC-NI & 2 & 7 & No & No & Yes \\
\hline $\begin{array}{l}\text { Rastogi } \text { et } \\
\text { al. }(20) \\
2017\end{array}$ & $\begin{array}{l}\text { Anesthesia, sterile saline solution } \\
\text { irrigation, PRF, and suture }\end{array}$ & HC-I & 4 & 2 & No & No & Yes \\
\hline $\begin{array}{l}\text { Chakra- } \\
\text { varthi }(9) \\
2017\end{array}$ & $\begin{array}{l}\text { Anesthesia, sterile saline solution } \\
\text { irrigation, PRF, and suture }\end{array}$ & HC-I & 4 & 2 & $\begin{array}{c}\text { Yes } \\
\text { Aceclofenac }\end{array}$ & No & Yes \\
\hline $\begin{array}{c}\text { Nikita } \text { et al. } \\
(21) \\
2016\end{array}$ & $\begin{array}{l}\text { Cleaning the socket with sterile } \\
\text { cotton pellets. Sterile saline } \\
\text { solution irrigation. Sterile cotton } \\
\text { mixed with pure nature honey }\end{array}$ & LC-NI & 3 & 4 & No & No & Yes \\
\hline \multirow{6}{*}{$\begin{array}{c}\text { Dubovina } \\
\text { et al. }(22) \\
2016\end{array}$} & $\begin{array}{l}\text { Sterile saline solution irrigation } \\
\text { and hyaluronic acid }\end{array}$ & LC-NI & 2 & 5 & No & No & No \\
\hline & \begin{tabular}{|l|} 
Sterile saline solution irrigation, \\
hyaluronic and aminocaproic acid
\end{tabular} & LC-NI & 2 & 5 & No & No & No \\
\hline & $\begin{array}{l}\text { Sterile saline solution irrigation } \\
\text { and Alveogyl® }\end{array}$ & LC-NI & 2 & 5 & No & No & No \\
\hline & $\begin{array}{l}\text { Anesthesia, curettage, and hyal- } \\
\text { uronic acid }\end{array}$ & LC-I & 3 & 5 & No & No & Yes \\
\hline & $\begin{array}{l}\text { Anesthesia, curettage, hyaluronic } \\
\text { and aminocaproic acid }\end{array}$ & LC-I & 3 & 5 & No & No & Yes \\
\hline & $\begin{array}{l}\text { Anesthesia, curettage, and Al- } \\
\text { veogyl@ }\end{array}$ & LC-I & 3 & 5 & No & No & No \\
\hline \multirow{3}{*}{$\begin{array}{l}\text { Rani et al. } \\
\quad(23) \\
2015\end{array}$} & $\begin{array}{l}\text { Anesthesia, sterile saline solution } \\
\text { irrigation and Alveogyl® }\end{array}$ & LC-NI & 3 & 1 & $\begin{array}{c}\text { Yes } \\
\text { Paracetamol }\end{array}$ & No & No \\
\hline & $\begin{array}{l}\text { Anesthesia, sterile saline solution } \\
\text { irrigation, and low-level diode } \\
\text { laser irradiation }\end{array}$ & HC-NI & 3 & 1 & $\begin{array}{c}\text { Yes } \\
\text { Paracetamol }\end{array}$ & No & Yes \\
\hline & $\begin{array}{l}\text { Anesthesia, sterile saline solution } \\
\text { irrigation, and low-level Er; Cr: } \\
\text { YSGG laser irradiation } \\
\end{array}$ & HC-NI & 3 & 1 & $\begin{array}{c}\text { Yes } \\
\text { Paracetamol }\end{array}$ & No & Yes \\
\hline \multirow{3}{*}{$\begin{array}{l}\text { Eshghpour } \\
\text { et al. }(24) \\
2015\end{array}$} & $\begin{array}{l}\text { Anesthesia, sterile saline solution } \\
\text { irrigation and Alveogyl@ }\end{array}$ & LC-NI & 3 & 4 & No & No & Yes \\
\hline & \begin{tabular}{|l|}
$\begin{array}{l}\text { Low power red InGaAlP laser } \\
\text { irradiation }\end{array}$ \\
\end{tabular} & HC-NI & 1 & 3 & No & No & Yes \\
\hline & $\begin{array}{l}\text { Low power infrared GaAlAs } \\
\text { laser irradiation }\end{array}$ & HC-NI & 1 & 3 & No & No & Yes \\
\hline \multirow{3}{*}{$\begin{array}{l}\text { Faizel et al. } \\
(25) \\
2014\end{array}$} & $\begin{array}{l}\text { Sterile saline solution irrigation } \\
\text { and Alveogyl } \AA\end{array}$ & LC-NI & 2 & 3 & No & No & Yes \\
\hline & $\begin{array}{l}\text { Sterile saline solution irrigation } \\
\text { and gauze piece soaked with zinc } \\
\text { oxide eugenol paste }\end{array}$ & LC-NI & 2 & 4 & No & No & No \\
\hline & $\begin{array}{l}\text { Sterile saline solution irrigation } \\
\text { and Neocones® }\end{array}$ & LC-NI & 2 & 2 & No & No & Yes \\
\hline $\begin{array}{l}\text { Singh } \text { et al. } \\
(26) \\
2014 \\
\end{array}$ & $\begin{array}{l}\text { Sterile gouge soaked with pure } \\
\text { nature honey }\end{array}$ & LC-NI & 1 & 7 & No & No & Yes \\
\hline \multirow{4}{*}{$\begin{array}{l}\text { Kaya et al. } \\
(27) \\
2011\end{array}$} & $\begin{array}{l}\text { Anesthesia, curettage, and sterile } \\
\text { saline solution irrigation }\end{array}$ & LC-I & 3 & 3 & No & No & No \\
\hline & \begin{tabular}{|l|} 
Anesthesia, curettage, sterile \\
saline solution irrigation and \\
Alveogyl ${ }^{\circledR}$ \\
\end{tabular} & LC-I & 4 & 3 & No & No & No \\
\hline & \begin{tabular}{|l} 
Anesthesia, curettage, sterile \\
saline solution irrigation, and \\
SaliCept Patch \\
\end{tabular} & LC-I & 4 & 3 & No & No & Yes \\
\hline & $\begin{array}{l}\text { Anesthesia, curettage, sterile } \\
\text { saline solution irrigation and low } \\
\text { level GaAlAs laser irradiation }\end{array}$ & HC-I & 4 & 3 & No & No & Yes \\
\hline
\end{tabular}

LC: low complexity; HC: high complexity; I: invasive; NI: non-invasive.

PRF: platelet rich fibrin. 
Sterile saline solution irrigation. Placement of PRF and suture. Anti-inflammatory analgesic medication with aceclofenac. Two treatment sessions required (9). This is the treatment that achieved the best pain control with fewer sessions required.

\section{Discussion}

Most of the current studies which address $\mathrm{AO}$ focus on the prevention and incidence reduction of this condition. Nonetheless, no consensus protocols on the treatment of $\mathrm{AO}$ were found, and choosing the best therapeutic option is still challenging for clinicians.

During the research and analysis of the consulted literature for this systematic review, a wide range of available treatments and variables were evident when evaluating therapeutic success. Consequently, we found several difficulties when comparing treatments. Pain is considered the most important symptom of AO which can vary in frequency and intensity leading the professional consultation (17). Thus, pain reduction was one of the clinical parameters considered for the analysis of these articles. An ideal AO treatment should get a faster remission of the intensity and duration of pain.

Regarding AO treatments analyzed in this study, those that showed the best performance used intra-alveolar irrigation (with sterile saline solution or iodopovidone) prior to other therapeutic procedures. Intra-alveolar irrigation offers many advantages such as microbial load reduction and necrotic tissue or clot debris removal. Almost all of the included studies used intra-alveolar irrigation as an early measure, complemented by other therapeutic approaches of different complexity. Interestingly, the use of intra-alveolar irrigation and curettage without complementary treatments showed poor results for pain control $(8,27)$. These results may indicate that intra-alveolar irrigation procedures are required, but not sufficient to obtain an acceptable decrease in pain.

Among the treatments which fulfilled the pain reduction proposed parameters, the most frequent procedure was an intra-alveolar placement of therapeutic products, excepting the use of low-level lasers or magnetotherapy. One of them, Alveogyl ${ }^{\circledR}$ (Septodont, Cambridge, Canada) is a therapeutic paste that contains iodoform (antiseptic), butamben (anesthetic), and eugenol (analgesic). Eugenol generates pain reduction through an inhibition mechanism of glutamatergic neurotransmission, activation of tumor necrosis factor-alpha (TNFa), and the endogenous opioid system (28). Neocones ${ }^{\circledR}$ (Septodont, Saint-Maur-des-Fossés, France), is a dental tablet that consists of polymyxin B sulfate (bactericidal on gram-negative), tyrothricin (bactericidal on gramnegative and spirochetes), and neomycin sulfate (broadspectrum antibiotic). Nevertheless, the pain reduction is mainly linked to a local anesthetic compound of tetracaine hydrochloride (25). SaliCept Patch ${ }^{\circledR}$ (Carrington,
Irving, USA), is a lyophilized product that contains an amorphous acemannan hydrogel (aloe vera plant filtrate). Acemannan gel is involved in macrophage activation, which stimulates fibroblast cytokine secretion and alveolar angiogenesis. TNF $\alpha$ and 1-interleukin are cytokines associated with anti-inflammatory effects and wound healing properties (29).

The aforementioned products (Alveogyl $\AA$, Neocones $\AA$, and Salicept Patch ${ }^{\circledR}$ ) were specifically developed for the treatment of AO. Nonetheless, PRF has been successfully developed for other therapeutic applications (30) and then used for AO treatment. PRF is obtained from a sample of patients' blood drawn at the moment of $\mathrm{AO}$ intervention. It does not require anticoagulants or platelet activators. PRF could control pain through a biologic mechanism linked to leukocyte functions and alveolar growth factors secretion such as transforming growth factor (TGF), 1-interleukin, fibroblast growth factor (FGF), platelet-derived growth factor (PDGF), and TNF $\alpha$. They increase and promote fibroblastic and angiogenic activity. Furthermore, the anti-nociceptive effects could be explained by the release of other substances like interleukins $(4,10,13)$, opioid peptides (endorphin beta, metencephalin, dynorphins), and insulin-like growth factor type 1 (IGF-1), which plays a fundamental role in cell growth (31).

Low-level laser therapy (LLLT) was a highly effective treatment that did not need to place an intra-alveolar therapeutic product. The wavelength of these lasers for dentistry use ranges from 635 to $950 \mathrm{~nm}$ (32). The mechanisms for LLLT-mediated pain relief are not fully understood. Several possible mechanisms are believed to explain the effects of LLLT, such as increased production of endogenous opioid neurotransmitters and local blood circulation by accelerating cellular redox reaction. An increased threshold for thermal pain as well as an increase in the production of adenosine triphosphate and anti-inflammatory cytokines (33). The advantages (25,31,34-36) and disadvantages (35-37) of each treatment are summarized in Table 4.

Only one of the analyzed treatments used antibiotic medication for AO management. It is widely accepted that systemic antibiotics do not have a greater advantage than local measures in immunocompetent patients $(5,38)$. On the other hand, considering that $\mathrm{AO}$ is a painful entity, analgesic-anti-inflammatory medication is a useful measure. Nevertheless, its use should not be considered more relevant than intra-alveolar procedures. The treatment developed by Chakravarthi (9), without overlooking that this was a non-randomized study with a critical risk of bias, showed one of the best pain control performances of the treatments included in this review. In the aforementioned study, $\mathrm{AO}$ patients were treated with an intra-alveolar placement of PRF and medicated with aceclofenac. 
Table 4: Advantages and disadvantages of alveolar osteitis treatments.

\begin{tabular}{|c|c|c|}
\hline Treatment & Advantages & Disadvantages \\
\hline Alveogyl ${ }^{\circledR}$ & $\begin{array}{l}\text { Bacteriostatic(25) } \\
\text { Hemostatic(36) } \\
\text { Low complexity }\end{array}$ & $\begin{array}{l}\text { Requires more sessions }(36) \\
\text { Possible foreign body reaction( } 36) \\
\text { Possible allergy to eugenol or iodine } \\
\text { Possible increased bone resorption. The material should be removed in the area } \\
\text { that receives future implants }\end{array}$ \\
\hline Neocones ${ }^{\circledR}$ & $\begin{array}{l}\text { Bactericide(25) } \\
\text { Low complexity }\end{array}$ & Possible allergy to derivatives of para-aminobenzoic acid and neomycin \\
\hline SaliCept Patch ${ }^{\circledR}$ & $\begin{array}{l}\text { Biocompatible(34) } \\
\text { Low complexity }\end{array}$ & Possible allergy to aloe vera \\
\hline $\begin{array}{c}\text { Low-level Diode } \\
\text { laser }\end{array}$ & $\begin{array}{l}\text { Bactericidal(35) } \\
\text { Hemostatic(35) } \\
\text { Minimally invasive }\end{array}$ & $\begin{array}{l}\text { High complexity } \\
\text { Risk of eye injury, protective glasses are recommended(35) } \\
\text { Use not recommended in patients with neoplasms, immunosuppressed, and blood } \\
\text { dyscrasias(35) }\end{array}$ \\
\hline $\begin{array}{l}\text { PRF (platelet rich } \\
\text { fibrin) }\end{array}$ & $\begin{array}{l}\text { Natural }(31) \\
\text { Highly biocompat- } \\
\text { ible(31) } \\
\text { Without preservatives } \\
\text { Minimal possibility } \\
\text { of adverse reactions }\end{array}$ & $\begin{array}{l}\text { High complexity } \\
\text { Morbidity at the injection site(37) } \\
\text { Use not recommended in chronic smokers, with alcohol and drug consumption, } \\
\text { immunocompromised, patients with acute and chronic infections, communicable } \\
\text { infectious diseases, sepsis, anticoagulants, chronic dermatological diseases, and } \\
\text { blood dyscrasias such as thrombocytopenia and hemodynamic instability(37) }\end{array}$ \\
\hline
\end{tabular}

Thus, it could be determined that the use of PRF and aceclofenac showed a combined-synergic effect in pain reduction. Despite this, there are studies where aceclofenac was not effective in the control of postoperative pain $(39,40)$, suggesting that pain reduction could be mainly due to intra-alveolar placement of PRF.

- Feasibility of clinical application

All AO treatments analyzed in this systematic review achieved pain reduction, although, with different time intervals, this is why all the treatments could potentially be applicable. The feasibility of clinical application of the best performance treatments should be considered according to their advantages and disadvantages (Table 4), operator training, equipment required for application, and cost-effectiveness analysis, among other variables. - Limitations of the present review

This study focuses on pain remission in AO treatment. However, other important variables such as epithelial healing, bone exposition, and analysis of adverse effects, were not considered.

Further RCTs are needed in order to validate the best performance AO treatments analyzed in this systematic review. These studies should also consider other features such as a combination of intra-alveolar procedures, pharmacological schemes, risk factors for $\mathrm{AO}$, and the record of clinical improvement variables (epithelial healing, bone exposition, necrotic debris, etc). Pain is a subjective experience, which means that it cannot be directly observed by those who are not experiencing it. This subjectivity generates a bias that is difficult to correct, since it is mainly due to the past experiences of individuals. This bias due to subjectiv- ity conditions the quality of the trials. In future studies, emphasis should be placed on reducing the impact of subjectivity by controlling the pre-intervention and measurement of outcomes domains.

\section{Conclusions}

AO treatment could be categorized into basic (intraalveolar irrigation) and specific procedures. The first ones should always be applied, and the second ones allow pain control success. There are invasive or noninvasive specific procedures, low or high complexity for the management of AO. The availability and the advantages or disadvantages of each could influence the selection of the therapeutic option.

\section{References}

1. Burgoyne CC, Giglio JA, Reese SE, Sima AP, Laskin DM. The efficacy of a topical anesthetic gel in the relief of pain associated with localized alveolar osteitis. J Oral Maxillofac Surg. 2010;68:144-8.

2. Blondeau F, Daniel NG. Extraction of impacted mandibular third molars: postoperative complications and their risk factors. J Can Dent Assoc. 2007;73:325

3. Taberner-Vallverdú M, Nazir M, Sánchez-Garcés MÁ, Gay-Escoda C. Efficacy of different methods used for dry socket management: A systematic review. Med Oral Patol Oral Cir Bucal. 2015;20:633-9.

4. Blum IR. Contemporary views on dry socket (alveolar osteitis): a clinical appraisal of standardization, aetiopathogenesis and management: a critical review. Int J Oral Maxillofac Surg. 2002;31:309-17.

5. Kolokythas A, Olech E, Miloro M. Alveolar Osteitis: A comprehensive review of concepts and controversies. Meurman JH, editor. Int J Dent. 2010;2010:249073.

6. Taberner-Vallverdú M, Sánchez-Garcés MÁ, Gay-Escoda C. Efficacy of different methods used for dry socket prevention and risk factor analysis: A systematic review. Med Oral Patol Oral Cir Bucal. 2017;22:750-8.

7. Macgregor AJ. Treatment of dry socket with zinc oxide and oil of 
cloves: Clinical Impressions. J Oral Ther Pharmacol. 1964;1:282-9. 8. Kamal A, Salman B, Abdul Razak NH, Qabbani AA, Samsudin AR. The efficacy of concentrated growth factor in the healing of alveolar osteitis: A Clinical Study. Int J Dent. 2020;2020:9038629.

9. Chakravarthi S. Platelet rich fibrin in the management of established dry socket. J Korean Assoc Oral Maxillofac Surg. 2017;43:160-5. 10. Moher D, Shamseer L, Clarke M, Ghersi D, Liberati A, Petticrew M, et al. Preferred reporting items for systematic review and meta-analysis protocols (PRISMA-P) 2015 statement. Systematic Reviews. 2015;1:4-1.

11. Higgins JPT, Altman DG, Gøtzsche PC, Jüni P, Moher D, Oxman $\mathrm{AD}$, et al. The Cochrane Collaboration's tool for assessing risk of bias in randomised trials. BMJ. 2011;343:d5928.

12. Sterne JA, Hernán MA, Reeves BC, Savović J, Berkman ND, Viswanathan M, et al. ROBINS-I: a tool for assessing risk of bias in non-randomised studies of interventions. BMJ. 2016;355:i4919.

13. Çebi AT. Evaluation of the effects of intra-alveolar irrigation with clindamycin, rifampicin and sterile saline in alveolar osteitis treatment. Journal of Stomatology, J Oral Maxillofac Surg. 2020;121:680-3. 14. Suchánek J, Ivančaková RK, Mottl R, Browne KZ, Pilneyová $\mathrm{KC}$, Pilbauerová N, et al. Hyaluronic acid-based medical device for treatment of alveolar osteitis-clinical Study. Int J Environ Res Public Health. 2019;16:3698.

15. Yüce E, Kömerik N. Potential effects of advanced platelet rich fibrin as a wound-healing accelerator in the management of alveolar osteitis: A randomized clinical trial. Niger J Clin Pract. 2019;22:1189-95. 16. King EM, Cerajewska TL, Locke M, Claydon NCA, Davies M, West NX. The efficacy of plasma rich in growth factors for the treatment of alveolar osteitis: A Randomized Controlled Trial. J Oral Maxillofac Surg. 2018;76:1150-9.

17. Supe NB, Choudhary SH, Yamyar SM, Patil KS, Choudhary AK, Kadam VD. Efficacy of alvogyl (combination of iodoform + butylparaminobenzoate) and zinc oxide eugenol for dry Socket. Ann Maxillofac Surg. 2018;8:193-9.

18. Chaurasia NK, Upadhyaya C, Dixit S. Comparative study to determine the efficacy of zinc oxide eugenol and alveogyl in treatment of dry socket. Kathmandu Univ Med J. 2017;15:203-6.

19. Lone PA, Ahmed SW, Prasad V, Ahmed B. Role of turmeric in management of alveolar osteitis (dry socket): A randomised clinical study. J Oral Biol Craniofac Res. 2018;8:44-7.

20. Rastogi S, Choudhury R, Kumar A, Manjunath S, Sood A, Upadhyay $\mathrm{H}$. Versatility of platelet rich fibrin in the management of alveolar osteitis-A clinical and prospective study. J Oral Biol Craniofac Res. 2018;8:188-93.

21. Soni N, Singh V, Mohammad S, Singh RK, Pal US, Singh R, et al. Effects of honey in the management of alveolar osteitis: A study. Natl J Maxillofac Surg. 2016;7:136-47.

22. Dubovina D, Mihailović B, Bukumirić Z, Vlahović Z, Miladinović M, Miković N, et al. The use of hyaluronic and aminocaproic acid in the treatment of alveolar osteitis. Vojnosanit Pregl. 2016;73:1010-5.

23. Rani A, Mohanty S, Sharma P, Dabas J. Comparative evaluation of Er:Cr:YSGG, diode laser and alvogyl in the management of alveolar osteitis: A prospective randomized clinical study. J Maxillofac Oral Surg. 2016;15:349-54.

24. Eshghpour M, Ahrari F, Najjarkar N-T, Khajavi M-A. Comparison of the effect of low level laser therapy with alvogyl on the management of alveolar osteitis. Med Oral Patol Oral Cir Bucal. 2015;20:386-92.

25. Faizel S, Thomas S, Yuvaraj V, Prabhu S, Tripathi G. Comparision between neocone, alvogyl and zinc oxide eugenol packing for the treatment of dry socket: a double blind randomised control trial. J Maxillofac Oral Surg. 2015;14:312-20.

26. Singh V, Pal US, Singh R, Soni N. Honey a sweet approach to alveolar osteitis: A study. Natl J Maxillofac Surg. 2014;5:31-4.

27. Kaya GŞ, Yapici G, Savaş Z, Güngörmüş M. Comparison of alvo- gyl, SaliCept patch, and low-level laser therapy in the management of alveolar osteitis. J Oral Maxillofac Surg. 2011;69:1571-7.

28. Bó WD, Luiz AP, Martins DF, Mazzardo-Martins L, Santos ARS. Eugenol reduces acute pain in mice by modulating the glutamatergic and tumor necrosis factor alpha (TNF- $\alpha$ ) pathways. Fundam Clin Pharmacol. 2013;27:517-25.

29. Zhang L, Tizard IR. Activation of a mouse macrophage cell line by acemannan: the major carbohydrate fraction from Aloe vera gel. Immunopharmacology. 1996;35:119-28.

30. Karimi K, Rockwell H. The benefits of platelet-rich fibrin. Facial Plast Surg Clin North Am. 2019;27:331-40.

31. Dohan Ehrenfest DM, Andia I, Zumstein MA, Zhang CQ, Pinto NR, Bielecki T. Classification of platelet concentrates (Platelet-Rich Plasma-PRP, Platelet-Rich Fibrin-PRF) for topical and infiltrative use in orthopedic and sports medicine: current consensus, clinical implications and perspectives. Muscles Ligaments Tendons J. 2014;4:3-9.

32. Luke AM, Mathew S, Altawash MM, Madan BM. Lasers: A review with their applications in oral medicine. J Lasers Med Sci. 2019;10:324-9.

33. Cotler HB, Chow RT, Hamblin MR, Carroll J. The use of low level laser therapy (LLLT) for musculoskeletal Pain. MOJ Orthop Rheumatol. 2015;2:00068.

34. Poor MR, Hall JE, Poor AS. Reduction in the incidence of alveolar osteitis in patients treated with the SaliCept patch, containing Acemannan hydrogel. J Oral Maxillofac Surg. 2002;60:374-9.

35. Kathuria V, Dhillon JK, Kalra G. Low level laser therapy: A panacea for oral maladies. Laser Ther. 2015;24:215-23.

36. AbdullGaffar B, Awadhi F. Be aware of a potential pitfall in oral and dental specimens: Alvogyl Fibers. Int J Surg Pathol. 2020;28:280-3.

37. Reddy SHR, Reddy R, Babu NC, Ashok GN. Stem-cell therapy and platelet-rich plasma in regenerative medicines: A review on pros and cons of the technologies. J Oral Maxillofac Surg Med Pathol. 2018:22:367

38. Bowe DC, Rogers S, Stassen LFA. The management of dry socket/alveolar osteitis. J Ir Dent Assoc. 2011;57:305-10.

39. Seymour RA, Frame J, Negus TW, Hawkesford JE, Marsden J, Matthew IR. The comparative efficacy of aceclofenac and ibuprofen in postoperative pain after third molar surgery. Br J Oral Maxillofac Surg. 1998;36:375-9.

40. Jain N, Maria A. Randomized double blind comparative study on the efficacy of Ibuprofen and aceclofenac in controlling post-operative sequelae after third molar surgery. J Maxillofac Oral Surg. 2011;10:118-22.

\section{Funding}

The authors declare no funding grants.

\section{Conflict of interest}

The authors declare that there is no conflict of interest with respect to the authorship and/or publication of this article.

\section{Ethics}

Udam sae coneculpa eariost, accae. Nam sunt, sent, num ut mo moluptatem resecta sunt.

\section{Authors contributions}

Garola Federico: Conceptualization, formal analysis, investigation, collection of data, and writing original draft.

Gilligan Gerardo: Conceptualization, formal analysis, investigation, and writing original draft.

Panico René: Supervision, review, and editing draft.

Leonardi Nicolás: Software, review, and editing draft.

Piemonte Eduardo: Investigation, software, supervision, review, and editing draft. 